\title{
MORTALIDADE INFANTIL NO NORDESTE BRASILEIRO: 2000 A
}

\section{4}

LIMA, A.S.R. ${ }^{1}$; COELHO, M.M.F. ${ }^{2}$; SILVA, C.F. ${ }^{3}$ \& MENEZES, L.C.G. ${ }^{4}$

${ }^{1}$ Enfermeira. Especialista em enfermagem pediátrica e neonatal pela Faculdade Metropolitana da Grande Fortaleza (FAMETRO). E-mail: ahellensaarah@ hotmail.com; ${ }^{2}$ Enfermeira. Doutora em Cuidados Clínicos em Enfermagem e Saúde pela UECE. Professora Adjunta do

Departamento de Enfermagem da Universidade Federal do Ceará (UFC). E-mail: manumfc2003@ yahoo.com.br; ${ }^{3}$ Enfermeira. Doutora em Saúde Coletiva e Mestre em Saúde Pública pela Universidade Federal do Ceará (UFC). Docente Titular do Centro Universitário Fametro

(UNIFAMETRO). E-mail: silva_ferreira_cristiana@yahoo.com.br; ${ }^{4}$ Enfermeira. Mestre e Doutora em Cuidados Clínicos em Enfermagem e Saúde pela UECE. Docente Titular do Centro Universitário Fametro (UNIFAMETRO). E-mail: lucianacatundagomes@ yahoo.com.br.

DOI: https://doi.org/10.32356/exta.v2.n18.31109 - Artigo submetido em 10/01/2018

\section{RESUMO}

O estudo analisou a mortalidade infantil na região Nordeste do Brasil no período de 2000 a 2014. Tratase de um estudo documental, descritivo e de abordagem quantitativa das taxas de mortalidade infantil. Foram utilizados dados do Sistema de Informação de Mortalidade e Sistema de Informações sobre Nascidos Vivos. Através das informações foram calculadas as taxas anuais de mortalidade infantil, de seus componentes (precoce, tardio e pós-neonatal) e a proporção das mortes por grupos de causas evitáveis. No período de 2000 a 2014 foram notificados 255.823 óbitos em menores de um ano no Nordeste, dos quais 168.653 (65,9\%) neonatais, $86.745(33,9 \%)$ pós-neonatais e 425 $(0,2 \%)$ óbitos com faixa etária ignorada. Houve uma redução da mortalidade no período estudado em todos os componentes, entretanto o componente neonatal precoce deteve o maior número de mortes,
\end{abstract}

correspondendo a 79,9\% dos óbitos neonatais. A taxa de mortalidade no Nordeste apresenta queda de $45,3 \%$ passando de 26,5 por mil nascidos vivos, em 2000 , para 14,5 mortes por mil nascidos vivos em 2014. O coeficiente de mortalidade proporcional por grupo de evitabilidade apresentou valores elevados entre as causas evitáveis registrando $70,1 \%$ dos óbitos, especialmente por adequada atenção à mulher na gestação, parto e ao recém-nascido $(\mathrm{RN})$. As causas mal-definidas e demais causas (não claramente evitáveis) representaram 10,3\% e 19,7\%, respectivamente, no período estudado. O Nordeste registrou grandes avanços na redução da mortalidade infantil nos últimos anos, tais mudanças podem estar associadas à intensificação de políticas públicas, programas e ações voltadas à atenção maternoinfantil.

PALAVRAS-CHAVE: Mortalidade Infantil. Serviço de Saúde Materno-Infantil. Epidemiologia.

\section{ATELIÊ DO IPREDE: EXPERIENCE AND ESTHETIC EDUCATION IN THE THIRD SECTOR}

\section{ABSTRACT}

The study examined infant mortality in the Northeast of Brazil from 2000 to 2014. This is a documental, descriptive, and quantitative approach to infant mortality rates. Data from the Mortality Information System and Information System on Live Births were used. Through the information, the annual rates of infant mortality, its components (precocious, late and post-neonatal) and the proportion of deaths by avoidable causes groups were calculated. Between 2000 and 2014, 255,823 deaths were reported in children under one year of age in the Northeast, of which $168,653(65.9 \%)$ were neonates, 86,745 $(33.9 \%)$ were post-neonatal, and $425(0.2 \%)$ ignored age. There was a reduction in mortality in the studied period in all components, however the early neonatal component had the highest number of deaths, orresponding to $79.9 \%$ of neonatal deaths. The mortality rate in the Northeast shows a $45.3 \%$ drop from 26.5 per thousand live births in 2000 to 14.5 deaths per thousand live births in 2014. The proportional mortality coefficient per avoidability group presented high values among avoidable causes, accounting for $70.1 \%$ of deaths, especially due to adequate attention to women in gestation, parturition and newborn. Poorly defined causes and other causes (not clearly avoidable) represented $10.3 \%$ and $19.7 \%$, respectively, in the studied period. The Northeast has made great progress in reducing child mortality in recent years. Such changes may be associated with the intensification of public policies, programs and actions directed at maternal and child care.

KEYWORDS: Infant Mortality. Maternal and Child Health Service. Epidemiology. 


\section{INTRODUÇÃO}

As estatísticas de mortalidade infantil representam um dos indicadores mais comumente utilizados para análise da situação de saúde de um país (WHO, 2014; BRASIL, 2013). Globalmente, a taxa de mortalidade em menores de cinco anos caiu de 91 mortes por mil nascidos vivos em 1990 para 43 mortes por mil nascidos vivos em 2015 (redução de 53\%). O Brasil reduziu de 60,8 óbitos por mil nascidos vivos para 16,4 no mesmo período (declínio de 73\%). Em 2011, o Brasil já havia alcançado a Meta do quarto Objetivo do Desenvolvimento do Milênio (ODM), quatro anos antes do prazo assumido perante a Organização das Nações Unidas (BRASIL, 2015a; DUTRA et al., 2015).

As taxas de mortalidade infantil (TMI) foram significativamente reduzidas. Desde 2000, o Brasil vem desenvolvendo políticas públicas sociais e econômicas voltadas para alcançar a redução da mortalidade em menores de cinco anos. E as ações resultaram em avanços nas condições de saúde, as quais são decorrentes da ampliação da cobertura da atenção básica, do acesso à vacinação, da elevação da taxa de aleitamento materno e do nível de escolaridade da mãe, além da diminuição da pobreza obtida pelo Programa Bolsa Família (PBF) (BRASIL, 2015a, 2015b).

O PBF é um programa de transferência condicionada de renda lançado em 2003, o qual tem desempenhado um papel significativo na redução da mortalidade infantil, geral e por causas relacionadas com a pobreza, como desnutrição e diarreia, nos municípios brasileiros nos últimos anos. Além disso, o programa reduziu substancialmente as taxas de hospitalização em menores de cinco anos, aumentou a cobertura vacinal e as consultas pré-natais, aumentando o primeiro contato com o sistema de saúde (RASELLA, 2013).

Apesar do notório decréscimo observado, parte considerável da mortalidade infantil é potencialmente evitável e sua distribuição desigual entre as regiões ainda indica a necessidade de intensificar ações voltadas para modificar este cenário, especialmente entre grupos sociais menos favorecidos (GONÇALVES et al., 2015).

O Nordeste por muito tempo apresentou altas TMI, mas com os avanços nas condições de saúde, em 2013, foi à região que apresentou o maior percentual de queda no Brasil com risco de mortalidade neonatal de 16,9 por mil nascidos (BRASIL, 2015c).

Diante do exposto, sobressai-se a necessidade de investigar a mortalidade infantil na região Nordeste do Brasil no período de 2000 a 2014, analisando a evolução ocorrida nas taxas de mortalidade ao longo de 15 anos depois de firmado o compromisso com as Nações Unidas 
para o ODM.

Assim, espera-se que o estudo possa se representar como uma importante ferramenta para identificação dos períodos de auge e declínio das TMI no Nordeste, especialmente após a instituição de ações e estratégias para melhoria da atenção à saúde da gestante, parturiente e ao recém-nascido. O presente estudo objetiva descrever a mortalidade infantil e suas principais causas na região Nordeste em 2000 a 2014.

\section{MORTALIDADE INFANTIL NA REGIÃO NORDESTE}

A mortalidade infantil é dividida em dois componentes: o neonatal, o qual estima os óbitos ocorridos nos primeiros 27 dias de vida, que se subdivide em neonatal precoce (em menores de sete dias) e tardio (entre sete e 27 dias), e o pós-neonatal, incluindo os óbitos ocorridos entre 28 dias até um dia antes de completar um ano (FERNANDES; VIEIRA; SCOCHI, 2013; LISBOA et al., 2015).

Os óbitos ocorridos em nosso país vêm se concentrando nos primeiros 27 dias de vida. Em 2015, o Brasil apresenta taxas da mortalidade do componente neonatal de 9,38 óbitos e o pós-neonatal de 5,72 por mil nascidos vivos (GBD, 2016).

Ao longo dos anos os óbitos em menores de um ano apresentaram mudanças no seu perfil segundo seus componentes em todas as regiões. No início dos anos 1990 as mortes se concentravam no componente pós-neonatal (51\% da mortalidade infantil) e a partir dos anos 2000 o componente neonatal precoce ganhou cada vez mais importância, representando mais de 50\% dos óbitos infantis nas regiões do Brasil (BRASIL, 2015c).

No entanto, essa evolução não ocorreu igualmente em algumas regiões do país. Em 1990, o Sul, Sudeste e Centro-Oeste já apresentavam proporções de óbitos pós-neonatal inferiores a $50 \%$, enquanto o Norte e no Nordeste se encontravam em torno de $60 \%$. A expressiva queda das taxas a partir de 1990 deveu-se à redução do componente pós-neonatal em todas as regiões, mas principalmente, nas regiões Norte e Nordeste ( $81 \%$ e $90 \%$, respectivamente) (BRASIL, 2015c).

A forte queda da mortalidade infantil no Nordeste desde 1990 implicou redução das diferenças desse indicador entre as regiões do país e deixa de apresentar as mais elevadas taxas desde meados de 2000. Nesse período o risco de morte era aproximadamente o dobro do encontrado no restante do país (BRASIL, 2011, 2015c).Em decorrência dos contrastes das taxas e desigualdade em saúde entre as regiões geográficas, o Governo Brasileiro implementou o Pacto pela Redução da Mortalidade Infantil na região Nordeste e Amazônia Legal, em 2009, 
visando diminuir as desigualdades regionais (GUERRERO, 2010; BRASIL, 2010).

A redução dessas taxas depende das mudanças nas condições vida e das ações definidas pelas políticas públicas de saúde, priorizando a gestação e nascimento na atenção a saúde da população (ROCHA, 2013).

\section{MÉTODO}

Trata-se de um estudo documental, descritivo e de abordagem quantitativa sobre a mortalidade infantil entre os nascidos vivos por local de ocorrência na região Nordeste. Os dados foram obtidos a partir de fonte secundária através do Sistema de Informações sobre Mortalidade (SIM) e Sistema de Informações sobre Nascidos Vivos (SINASC). Foram inclusos os óbitos ocorridos entre 01 de janeiro de 2000 a 31 de dezembro de 2014.

A seleção do período, 2000 a 2014, ocorreu pela disponibilidade dos dados no Departamento de Informática do Sistema Único de Saúde no momento da coleta e pelo ano completo disponível. Não foi necessária a submissão do presente estudo ao Comitê de Ética de Pesquisas em Seres Humanos por se tratar de dados públicos e de livre acesso disponíveis nos sistemas de informações do Ministério da Saúde.

O levantamento de dados foi realizado no período de janeiro de 2017. Para coleta de dados dos Sistemas de Informações do Ministério da Saúde, foram gerados arquivos do próprio sistema em formato de tabelas. As informações foram compiladas no programa Microsoft Excel 2007. Neste programa foram calculadas as taxas anuais de mortalidade infantil e de seus componentes (precoce, tardio e pós-neonatal) por 1000 mil nascidos vivos e a proporção das mortes por grupos de causas evitáveis a cada triênio, e distribuição percentual dos óbitos infantis pela causa básica de morte através da Classificação Internacional de Doenças 10 (CID10). Os dados foram submetidos à análise descritiva e analítica, agrupados em tabelas e analisados à luz da literatura.

No Brasil, o critério de morte por evitabilidade é dependente de tecnologia disponível em nosso país, de tecnologia acessível pela maior parte da população brasileira ou de tecnologia ofertada pelo Sistema Único de Saúde (SUS). Em 2007, a partir de diretrizes estabelecidas, da comparação das listas de referência de Ortiz e de Tobias \& Jackson em função da revisão realizada por Malta e Duarte foi proposta a lista de morte evitáveis segundo grupo etário: para menores de cinco anos de idade e para faixa etária de cinco a 75 anos. (MALTA et al., 2007).

A lista de causa de mortes evitáveis para menores de cinco anos de idade segundo Malta e Duarte abrange as reduzíveis por: ações de imunoprevenção; adequada atenção à mulher na 
gestação e parto e ao recém-nascido; ações adequadas de diagnóstico e tratamento; ações adequadas de promoção à saúde, ações adequadas de atenção à saúde. (MALTA et al., 2007).

\section{RESULTADOS}

No período de 01 de janeiro de 2000 a 31 de dezembro 2014 foram notificados 255.823 óbitos em menores de um ano no Nordeste, dos quais $168.653(65,9 \%)$ neonatais, 86.745 $(33,9 \%)$ pós-neonatais e $425(0,2 \%)$ óbitos com faixa etária ignorada. No SINASC nesse mesmo período foram notificados 13.266 .492 nascidos vivos. A figura 1 apresenta o coeficiente de mortalidade infantil por componente na região Nordeste. Observa-se a redução da mortalidade no período estudado em todos os componentes. O componente neonatal precoce deteve o maior número de mortes durante todo o período estudado.

FIGURA 1 - ÓBITOS INFANTIS POR COMPONENTE NA REGIÃO NORDESTE, BRASIL, 2000 A 2014.

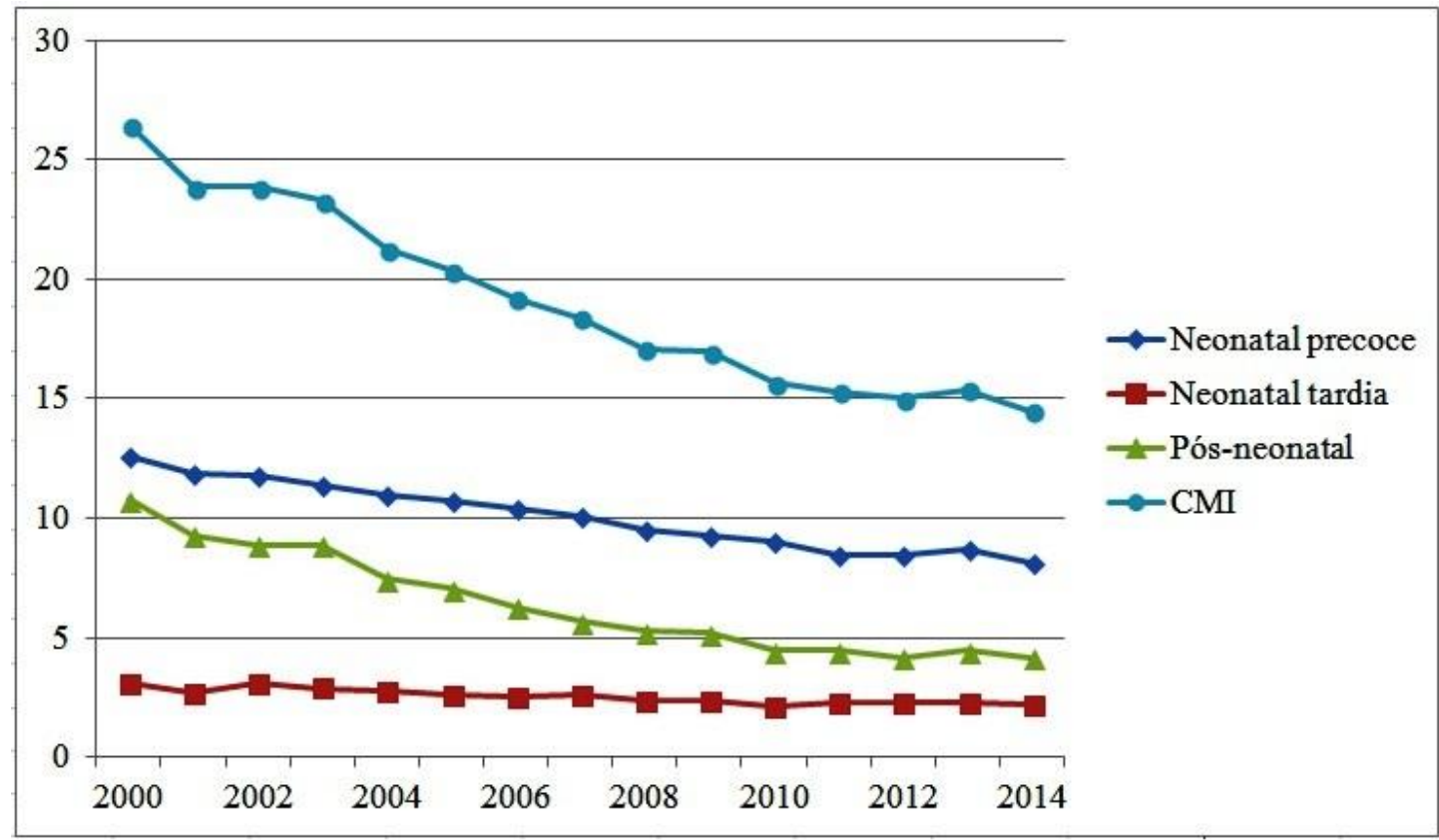

Fonte: SINASC e SIM

A tabela 1 apresenta o coeficiente de mortalidade infantil entre as Unidades Federativas (UF) da região Nordeste. Observa-se uma tendência de queda nos óbitos em menores de um ano nessa região, especialmente em Paraíba, Alagoas e Pernambuco os quais apresentaram maior variação entre os demais estados. 


\begin{tabular}{|c|c|c|c|c|c|c|c|c|c|c|c|c|c|c|c|}
\hline \multirow[b]{2}{*}{ UF } & \multicolumn{15}{|c|}{ Coeficiente de mortalidade infantil anual } \\
\hline & $\underset{8}{8}$ & ర్రి & ণ্ণ & §ి & ষ্ণ & 苂 & ঠి & 요 & 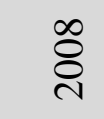 & ஓి & $\stackrel{\circ}{\circ}$ & $\bar{\Xi}$ & $\stackrel{ }{\stackrel{ }{\delta}}$ & $\stackrel{m}{\stackrel{n}{i}}$ & $\frac{ \pm}{\grave{D}}$ \\
\hline MA & 18,1 & 19,3 & 19,6 & 18,4 & 6,8 & ,2 & 9 & 4 & 15,8 & 15,9 & 15,1 & 15,7 & 14,4 & 16,1 & 5,3 \\
\hline PI & 23,4 & 22,6 & 23,9 & 23,8 & 21,1 & 21,5 & 21,0 & 21,4 & 20,2 & 19,6 & 17,9 & 17,8 & 17,5 & 17,3 & 16,0 \\
\hline $\mathrm{CE}$ & 26,8 & 21,4 & 24,4 & 25,3 & 22,7 & 18,5 & 18,2 & 16,1 & 15,7 & 15,5 & 13,1 & 13,8 & 12,7 & 13,9 & 12,3 \\
\hline $\mathrm{RN}$ & 21,2 & 19,2 & 20,9 & 17,3 & 18,3 & 16,3 & 14,5 & 15,5 & 14,4 & 13,7 & 13,1 & 13,1 & 13,8 & 14,2 & 12,6 \\
\hline PR & 32,3 & 21,0 & 23,2 & 21,9 & 20,9 & 18,9 & 18,1 & 18,5 & 16,6 & 14,8 & 14,5 & 14,1 & 14,3 & 14,3 & 13,3 \\
\hline PE & 30,2 & 27,0 & 26,1 & 26,6 & 22,9 & 21,7 & 19,1 & 19,4 & 17,6 & 18,0 & 16,0 & 15,0 & 15,2 & 15,0 & 1 \\
\hline $\mathrm{AL}$ & 31,4 & 31,8 & 29,7 & 26,9 & 24,2 & 23,8 & 21,2 & 21,0 & 18,0 & 18,6 & 16,7 & 15,6 & 15,2 & 16,1 & 15,2 \\
\hline SE & 30,3 & 33,3 & 29,5 & 27,6 & 24,2 & 25,8 & 22,7 & 19,8 & 19,0 & 17,8 & 16,0 & 16,6 & 16,8 & 15,7 & 15,8 \\
\hline BA & 25,9 & 23,7 & 22,7 & 22,6 & 21,7 & 21,2 & 21,1 & 19,1 & 17,9 & 18,0 & 17,4 & 16,0 & 16,1 & 16,3 & 15,5 \\
\hline $\mathrm{NE}$ & 26,5 & 23,9 & 23,9 & 23,3 & 21,3 & 20,4 & 19,2 & 18,4 & 17,1 & 17,0 & 15,7 & 15,3 & 15,0 & 15,4 & 14,5 \\
\hline
\end{tabular}

Na tabela 2 são apresentadas as taxas de mortalidade infantil (TMI) e mortalidade proporcional durante os cinco triênios segundo a classificação de evitabilidade de Malta et al. Entre 2000 a 2014, verifica-se que 70,1\% dos óbitos foram classificados como evitáveis, principalmente por adequada atenção à mulher na gestação, parto e ao recém-nascido (RN). As causas mal-definidas e demais causas (não claramente evitáveis) representaram 10,3\% e 19,6\%, respectivamente, no período estudado. 
TABELA 2 - TMI E MORTALIDADE PROPORCIONAL (\%) POR PRINCIPAIS GRUPOS DE CAUSAS SEGUNDO CRITÉRIO DE EVITABILIDADE NA REGIÃO NORDESTE, BRASIL, 2000 A 2014.

\begin{tabular}{|c|c|c|c|c|c|c|c|c|c|c|}
\hline \multirow{2}{*}{ Categorias } & \multicolumn{2}{|c|}{$2000-2002$} & \multicolumn{2}{|c|}{ 2003-2005 } & \multicolumn{2}{|c|}{ 2006-2008 } & \multicolumn{2}{|c|}{ 2009-2011 } & \multicolumn{2}{|c|}{ 2012-2014 } \\
\hline & $\sum_{i}$ & $\%$ & $\sum_{i}$ & $\%$ & $\sum_{i}$ & $\%$ & $\sum_{i}$ & $\%$ & $\sum_{i}$ & $\%$ \\
\hline 1 Causas evitáveis & 16,5 & 66,6 & 15,2 & 70,1 & 13,3 & 72,7 & 11,5 & 71,8 & 10,7 & 71,3 \\
\hline $\begin{array}{l}1.1 \text { Ações de } \\
\text { imunoprevenção }\end{array}$ & 0,02 & 0,1 & 0,02 & 0,1 & 0,01 & 0,1 & 0,01 & 0,1 & 0,03 & 0,2 \\
\hline $\begin{array}{l}\text { 1.2 Adequada atenção na } \\
\text { gestação, parto e ao RN }\end{array}$ & 11,7 & 47,1 & 11,0 & 50,6 & 10,2 & 56 & 9,3 & 58 & 8,8 & 58,5 \\
\hline $\begin{array}{l}\text { 1.2.1 Adequada atenção à } \\
\text { mulher na gestação }\end{array}$ & 4 & 16,3 & 4,4 & 20,3 & 4,6 & 25,2 & 4,2 & 26 & 3,9 & 26 \\
\hline $\begin{array}{l}\text { 1.2.2 Adequada atenção à } \\
\text { mulher no parto }\end{array}$ & 2,3 & 9,5 & 2,3 & 10,6 & 2,3 & 12,3 & 2,0 & 12,4 & 1,8 & 12,1 \\
\hline $\begin{array}{l}\text { 1.2.3 Adequada atenção } \\
\text { ao RN }\end{array}$ & 5,3 & 21,4 & 4,3 & 19,7 & 3,4 & 18,4 & 3,1 & 19,5 & 3,1 & 20,4 \\
\hline $\begin{array}{l}\text { 1.3 Ações adequadas de } \\
\text { diagnóstico e tratamento }\end{array}$ & 2,0 & 8,0 & 1,8 & 8,5 & 1,4 & 7,8 & 1,2 & 7,4 & 1,1 & 7,1 \\
\hline $\begin{array}{l}1.4 \text { Ações de promoção à } \\
\text { saúde }\end{array}$ & 2,8 & 11,3 & 2,4 & 10,9 & 1,6 & 8,8 & 1,0 & 6,4 & 0,8 & 5,5 \\
\hline 2. Causas mal-definidas & 4,8 & 19,3 & 2,7 & 12,4 & 0,9 & 4,8 & 0,7 & 4,1 & 0,6 & 3,8 \\
\hline $\begin{array}{l}\text { 3. Demais causas (não } \\
\text { claramente evitáveis) }\end{array}$ & 3,5 & 14,1 & 3,8 & 17,6 & 4,1 & 22,4 & 3,8 & 24,1 & 3,7 & 24,9 \\
\hline Total das categorias & 24,7 & 100 & 21,7 & 100 & 18,2 & 100 & 16,0 & 100 & 15,0 & 100 \\
\hline
\end{tabular}

A tabela 3 apresenta a taxa dos óbitos infantis segundo a causa de morte por pelo capítulo do CID10. Observa-se que a maior da taxa dos óbitos evitáveis se concentra na atenção à mulher na gestação, parto e ao RN representando 75,6 dos casos e as principais causas estão relacionadas a algumas afecções originadas no período perinatal. 
TABELA 3 - DISTRIBUIÇÃO PERCENTUAL DOS ÓBITOS INFANTIS SEGUNDO CRITÉRIO DE EVITABILIDADE E CAPÍTULO DO CID 10 NA REGIÃO NORDESTE, BRASIL, 2000 A 2014.

\begin{tabular}{lcc}
\hline \multicolumn{1}{c}{ Causa de morte pelo capítulo do CID10 } & \multicolumn{2}{c}{ Mortalidade proporcional } \\
\cline { 2 - 3 } 1. Causas evitáveis & $\mathrm{n}$ & $\%$ \\
1.1 Ações de imunização & 179285 & 70,1 \\
Algumas doenças infecciosas e parasitárias & 275 & 0,1 \\
Doenças do sistema nervoso & 233 & 0,1 \\
Algumas afecções originadas no período perinatal & 11 & 0,0 \\
1.2. Atenção à gestação, parto, feto e recém-nascido & 31 & 0,0 \\
Algumas doenças infecciosas e parasitárias & 135612 & 53,0 \\
Algumas afecções originadas no período perinatal & 663 & 0,3 \\
1.3 Ações de diagnóstico e tratamento adequado & 134949 & 52,8 \\
Algumas doenças infecciosas e parasitárias & 20097 & 7,9 \\
Doenças do sistema nervoso & 6409 & 2,5 \\
Doenças do aparelho respiratório & 1061 & 0,4 \\
Doenças endócrinas, nutricionais e metabólicas & 11471 & 4,5 \\
Malformações congênitas, deformidades e anomalias & 93 & 0,0 \\
Doenças do aparelho geniturinário & 855 & 0,3 \\
Doenças do aparelho circulatório & 197 & 0,1 \\
1.4 Ações promoção à saúde & 11 & 0,0 \\
Algumas doenças infecciosas e parasitárias & 23301 & 9,1 \\
Doenças do sangue e dos órgãos hematopoiéticos & 13534 & 5,3 \\
Doenças endócrinas, nutricionais e metabólicas & 316 & 0,1 \\
Causas externas de morbidade & 5537 & 2,2 \\
Sintomas, sinais e achados anormais de exames clínicos & 2857 & 1,1 \\
2. Causas mal-definidas & 1057 & 0,4 \\
3. Demais causas & 26257 & 10,3 \\
\hline Total & 50281 & 19,6 \\
\hline
\end{tabular}

Nas ações de imunização as principais causas se relacionam a algumas doenças infecciosas e parasitárias, destacamos a coqueluche e tétano neonatal. No subgrupo de ações por diagnóstico e tratamento adequado verifica-se que as doenças do aparelho respiratório somadas às doenças infecciosas e parasitárias foram responsáveis pela maioria dos óbitos registrados, sobressaindo-se à pneumonia e a outras doenças bacterianas. Nas ações de promoção à saúde as principais causas relacionadas foram algumas doenças infecciosas e parasitárias e as doenças endócrinas, nutricionais e metabólicas, com maior destaque a desnutrição e outras deficiências nutricionais.

\section{DISCUSSÃO}

Os resultados desse estudo demonstram que a TMI na região Nordeste apresentou 
declínio no período de 2000 a 2014, especialmente no componente pós-neonatal (queda de 39,8\%). Desde 1990 a 2013, evidencia-se uma redução significativa em todas as regiões brasileiras em relação à mortalidade pós-neonatal, todavia a redução mais importante deste componente ocorreu no Nordeste, região historicamente conhecida por suas altas taxas. Isso pode ter sido resultado das ações como o Plano de Redução das Desigualdades na Mortalidade Infantil no Nordeste e Amazônia Legal, além de programas de transferência de renda como o Programa Bolsa Família (BRASIL, 2015c).

O Pacto pela Redução da Mortalidade Infantil no Nordeste e Amazônia Legal foi constituído através da Portaria $\mathrm{n}^{\circ} 364 / 2009$, a qual firmava o compromisso do governo federal para acelerar a redução das desigualdades nessas regiões. O objetivo da proposta era reduzir em 5\% ao ano a TMI nos anos de 2009 e 2010, com prioridade para o componente neonatal. As principais metas do Pacto incluíam a ampliação: das equipes de saúde da família e qualificação dos profissionais, dos leitos de unidade de tratamento intensivo e unidade de cuidados intermediários, dos bancos de leite humano, dos Hospitais Amigo da Criança e maternidades com equipes capacitadas para método canguru (GUERRERO, 2010; BRASIL, 2010).

Os programas de transferência de renda também representam uma estratégia bem concebida de redução da pobreza demonstrando serem eficazes no aumento da utilização dos serviços de saúde e terem um impacto positivo geral sobre vários desfechos de saúde, dentre eles a redução da mortalidade infantil (RASELLA, 2013).

O PBF é um programa de transferência de renda condicionado, o qual transfere para as famílias pobres um piso de consumo mínimo para amenizar os efeitos da pobreza e as suas condicionalidades são centradas na saúde e educação. As condicionalidades relacionadas à saúde são geralmente aplicadas às crianças de zero a seis anos e as mulheres grávidas, preconizando que as crianças visitem regularmente os centros de saúde, cumpram o calendário vacinal, monitorizem o crescimento e desenvolvimento, do mesmo modo preconiza as consultas adequadas durante o pré-natal. Ademais, favorece na nutrição ofertada à criança e gestante através do aumento do consumo de alimentos (RASELLA, 2013).

Dentre os nove estados da região Nordeste, quatro conseguiram uma redução de mais da metade dos óbitos ocorridos durante os 15 anos. O estado de Maranhão apresentou o menor percentual de redução, entretanto desde 2000 já atingia as menores taxas. Ressalta-se que é necessário considerar que o SIM apresenta subnotificação de óbito infantil, particularmente nas regiões Norte e Nordeste. Segundo o Instituto Brasileiro de Geográfica Estatística (IBGE) o 
SIM desde sua concepção apresenta subnotificações, todavia vem mostrado melhoras na cobertura em relação aos óbitos (RIPSA, 2008; SILVA, 2009).

Em 2008, o Ministério da Saúde deu início a uma série de medidas para acelerar a vigilância de óbitos no âmbito do SUS, identificando os determinantes dos óbitos em tempo oportuno. Nesse período a Secretaria de Vigilância à Saúde publicou portarias voltadas a vigilância dos óbitos infantis e maternos definindo o Norte e Nordeste como regiões estratégicas. A Portaria $\mathrm{n}^{\mathrm{o}}$ 1.119/2008 estabelece a vigilância do óbito materno obrigatória nos serviços do SUS. Outras portarias fundamentais para a estruturação de óbitos incluem: Portaria $\mathrm{n}^{\circ} 116 / 2009$, a qual trata sobre os fluxos e prazos para as notificações das mortes infantis, fetais e maternas; Portaria $n^{\circ} 72 / 2010$, que regulamenta a vigilância de óbitos infantil e fetal; Portaria $\mathrm{n}^{\mathrm{o}} 1.020 / 2013$, que trata das atribuições dos hospitais à gestante de alto risco e a realização da investigação de óbito infantil, fetal e materno (BRASIL, 2015c).

O Nordeste brasileiro apesar de apresentar desigualdades subnacionais é citado como uma região de potencial progresso em meio às disparidades ao longo do tempo, conseguindo registrar diminuição nas taxas de mortalidade em menores de cinco anos desde 2000 (GBD, 2016).

$\mathrm{Na}$ pesquisa também se observa que o componente neonatal precoce no Nordeste apresentou redução ao longo dos anos, entretanto demonstrando taxas elevadas, representandose como um problema de saúde pública. Pesquisas conduzidas em Minas Gerais identificaram tendência semelhante, as quais obtiveram redução nos componentes neonatal tardio e pósneonatais, porém elevação nos coeficientes de mortalidade neonatal precoce (MENEZES et al., 2014; LISBOA et al., 2015).

Globalmente as taxas de mortalidade neonatal também se apresentam com decréscimo mais lento, sobretudo no componente neonatal precoce (RASELLA, 2013). Em um estudo realizado em 194 países, evidenciou-se que no conjunto de dados de 65 países os óbitos neonatais precoces corresponderam a 75,8\% dos ocorridos (OZA et al., 2016).

A redução da TMI e suas causas ao longo dos triênios no presente estudo permite observar avanços nos últimos 15 anos. Entretanto, as proporções de mortes infantis por causas evitáveis ainda são muito elevadas, principalmente no subgrupo de adequada atenção à mulher na gestação, parto e ao RN, revelando desafios a serem superados na assistência materno-infantil. Se as mortes evitáveis por atenção à gestante no pré-natal e no parto e ao recém-nascido fossem de fato evitadas, a taxa de mortalidade neonatal se aproximaria da taxa de países desenvolvidos 
(NASCIMENTO; ALMEIDA; GOMES, 2014). Sabe-se que a morte evitável pode derivar de indicadores sensíveis à qualidade da atenção à saúde prestada pelo sistema de saúde, que, por sua vez, podem acarretar a tomada de medidas de resultado ou de impacto dessa atenção (MALTA et al., 2007).

No Brasil, são intensificados políticas públicas, programas e ações voltadas à atenção materno-infantil visando à qualidade na atenção da saúde da mulher durante o pré-natal, parto e atenção ao recém-nascido e a redução das taxas de mortalidade. Dentre eles podemos destacar o Programa de Humanização do Pré-Natal e do Nascimento, o Pacto Nacional pela Redução da Mortalidade Materna e Neonatal e o Programa Rede Cegonha (BRASIL, 2000, 2004, 2011; CASSIANO et al., 2014).

No grupo de causas evitáveis reduzíveis por adequada atenção à mulher na gestação, seguidas de adequada atenção à mulher no parto e adequada atenção ao RN apresentaram como principais responsáveis dos óbitos nessa pesquisa, respectivamente, a síndrome da angústia respiratória do RN, a hipóxia intrauterina e asfixia ao nascer, as infecções específicas do período neonatal. Estudos semelhantes observaram o mesmo padrão de causas de óbitos (LOURENÇO; BRUNKEN; LUPPI, 2013; MENEZES et al., 2014; LISBOA et al., 2015).

Com relação à categoria de ações por diagnóstico e tratamento adequado, verificou-se na pesquisa que o principal responsável pelos óbitos foi à pneumonia. E no grupo de causas redutíveis por ações adequadas de promoção à saúde se destacam as doenças infecciosas intestinais. Em uma pesquisa conduzida em 194 países membros da Organização Mundial da Saúde as causas registradas se assemelham as da região Nordeste. As principais causas de mortalidade neonatal estão associadas à prematuridade e complicações relacionadas ao nascimento, enquanto as principais causas de morte pós-neonatal foram pneumonia, diarreia, lesões e malária (BRASIL, 2015c; OZA et al., 2015; WHO, 2016).

As menores proporções de óbitos dessa pesquisa foram atribuídas ao grupo de causas por ações de imunoprevenção. O resultado registrado está associado ao avanço no acesso à vacinação através do Programa Nacional de Imunizações, ofertando vacinação desde o primeiro ano de vida contribuindo para a prevenção de doenças transmissíveis. Ressalta-se ainda, avanços na ampliação da cobertura da atenção primária através da Estratégia saúde da Família (AQUINO; OLIVEIRA; BARRETO, 2009; FRANÇA et al., 2009; BRASIL, 2015a). Também se destaca a implantação de programas direcionados ao incentivo do aleitamento materno, bem como a relativa expansão do sistema de saneamento básico (IBGE, 2010; BRASIL, 2015a). 
Reduzir a mortalidade neonatal exige uma melhor prevenção e gestão de partos prematuros, cuidados e suporte de recém-nascidos doentes, gestão de infecções graves e a promoção do cuidado através do método canguru. Para o período pós-neonatal, são necessários maiores esforços para ampliar a cobertura de novas vacinas e para melhorar a cobertura do tratamento para pneumonia e diarreia (WHO, 2016).

Países que atingiram a quarta meta do ODM destacam a importância de abordagens multissetoriais para melhorar a saúde infantil, como a implementação de políticas para alcançar o acesso universal a cuidados primários para mulheres e crianças, o aumento dos gastos gerais do governo em saúde materno-infantil e a implementação de programas que promover a educação e capacitação entre as mulheres (GBD, 2016).

O Brasil desempenhou papel fundamental na implementação dos ODM e tem mostrado empenho diante dos novos objetivos firmados com as Nações Unidas através de uma agenda mundial com os Objetivos de Desenvolvimento do Sustentável (ODS). Essa agenda global foi adotada em 2015 e assinada por 193 países, entre os quais o Brasil, contemplando 17 Objetivos e 169 metas envolvendo temáticas diversificadas, as quais deverão ser adotadas até 2030 (OPAS, 2016; BRASIL, 2017).

\section{CONCLUSÃO}

Os resultados deste estudo evidenciam que, na região Nordeste, a mortalidade infantil apresentou redução no período da pesquisa apresentando grande avanço entre as regiões do Brasil. A maioria dos óbitos é evitável, especialmente no subgrupo de adequada atenção à mulher na gestação, parto e ao RN. Esses achados são considerados aspectos importantes e devem ser realizados esforços com o objetivo de diminuir os óbitos evitáveis através da melhoria na assistência pré-natal, parto e nascimento, visando à qualidade da assistência ofertada ao binômio mãe-filho desde a atenção básica até atenção hospitalar.

A utilização da lista de causas de mortes evitáveis em menores de cinco anos é um instrumento que permite identificar os principais grupos com maiores taxas de óbitos e através dos dados obtidos planejar estratégias para redução da mortalidade infantil conforme a realidade de cada região.

Sugere-se que novos estudos sejam desenvolvidos em campo para obtenção de uma visão mais fidedigna referente às causas de óbito, haja vista o número de óbitos com faixa etária ignorada, além da possibilidade de subnotificação dos óbitos no SIM e SINASC.

Espera-se que os resultados apresentados neste estudo auxiliem no planejamento de ações 
direcionadas a redução da mortalidade infantil, possibilitando aperfeiçoar os cuidados oferecidos as gestantes, recém-nascido e lactente.

\section{REFERENCIAS}

AQUINO R; OLIVEIRA, N.F; BARRETO, M.L. Impact of the Family Health Program on infant mortality in Brazilian municipalities. Journal of Public Health American, v.99, n.1, p.87-93, 2009. Disponível em < https://www.ncbi.nlm.nih.gov/pmc/articles/PMC2636620/>. Acesso em: set. 2017.

BRASIL. Portaria $n^{\circ} 569$, de $1^{\circ}$ de junho de 2000. Institui o Programa de Humanização no Pré-natal e Nascimento, no âmbito do Sistema Único de Saúde. Ministério da Saúde, Brasília, 1 de junho de 2000. Disponível em < http://bvsms.saude.gov.br/bvs/saudelegis/gm/2000/prt0569_01_06_2000_rep.html>.Acesso em: jan. 2017.

. Ministério da saúde. Secretaria de atenção a saúde. Departamento de ações programáticas estratégicas. Pacto Nacional pela Redução da Mortalidade Materna e Neonatal, Brasília, 2004. Disponível em< http://www.saude.ba.gov.br/dab/Pacto_Aprovado_na_tripartite.pdf> Acesso em: jan. 2017.

Ministério da Saúde. Secretaria de Atenção à Saúde. Departamento de Ações Programáticas Estratégicas. Pacto pela redução da mortalidade infantil no Nordeste e Amazônia Legal: 2009-2010. Brasília, 2010. Disponível em < http://bvsms.saude.gov.br/bvs/publicacoes/pacto_reducao_mortalidade_infantil_nordeste.pdf> . Acesso em: mar. 2017.

Portaria ${ }^{\circ} 1.459$, de 24 de junho de 2011. Institui, no âmbito do Sistema Único de Saúde SUS a Rede Cegonha. Ministério da Saúde, Brasília, 24 de junho de 2011. Disponível em <http://bvsms.saude.gov.br/bvs/saudelegis/gm/2011/prt1459_24_06_2011.html>. Acesso em: dez. 2016.

Ministério da Saúde. Secretaria de Vigilância em Saúde. Departamento de Análise de Situação em Saúde. Brasil 2012: uma análise da situação de saúde e dos 40 anos do Programa Nacional de Imunizações, Brasília, 2013. Disponível em:

<http://bvsms.saude.gov.br/bvs/publicacoes/saude_brasil_2012_analise_situacao_saude.pdf >. Acesso em: jan. 2017.

Ministério da Saúde. Brasil reduz mortalidade na infância em 20\% acima da média mundial. Portal de Saúde, 02 out. 2015a. Disponível em <http://portalsaude.saude.gov.br/index.php/cidadao/principal/agencia-saude/19982-brasilreduz-mortalidade-na-infancia-em-20-acima-da-media-mundial>. Acesso em: jan. 2017. 
. Ministério da Saúde. ONU: Brasil cumpre meta de redução da mortalidade infantil. Portal de Saúde, 09 set. 2015b. Disponível em < http://www.brasil.gov.br/cidadania-ejustica/2015/09/onu-brasil-cumpre-meta-de-reducao-da-mortalidade-infantil >. Acesso em: 11 jan. 2017.

Ministério da Saúde. Secretaria de Vigilância em Saúde. Departamento de Vigilância de Doenças e Agravos Não Transmissíveis e Promoção da Saúde. Saúde Brasil 2014: uma análise da situação de saúde e das causas externas, Brasília, 2015c. Disponível em: $<$ http://bvsms.saude.gov.br/bvs/publicacoes/saude_brasil_2014_analise_situacao.pdf>. Acesso em: jan. 2017.

Ministério das Relações Exteriores. Objetivos de Desenvolvimento Sustentável. Itamaraty, 02 set. 2017. Disponível em <http://www.itamaraty.gov.br/pt-BR/politicaexterna/desenvolvimento-sustentavel-e-meio-ambiente/134-objetivos-de-desenvolvimentosustentavel-ods>. Acesso em: set. 2017.

CASSIANO, A. C. M. et al. Saúde materno infantil no Brasil: evolução e programas desenvolvidos pelo Ministério da Saúde. Revista do Serviço Público Brasília, v.65,n.2, p. 227-244, 2014. Disponível em < https://revista.enap.gov.br/index.php/RSP/article/view/581/499>. Acesso em: mar. 2017.

DUTRA, I. R. et al. Investigação dos óbitos infantil e fetal no Vale do Jequitinhonha, Minas Gerais, Brasil. Revista Mineira Enfermagem, v.19, n.3, p.597-604, 2015.

FERNANDES, C.A; VIEIRA, V.C.L; SCOCHI, M.J. Mortalidade infantil e classificação de evitabilidade: pesquisando municípios da 15 regional de saúde Paraná. Revista Ciência Cuidado e Saúde, v.12, n.4, p.752-759, 2013. Disponível em < http://www.periodicos.uem.br/ojs/index.php/CiencCuidSaude/article/view/16537>. Acesso em: mar. 2017.

FRANÇA, I.S.X. et al. Cobertura vacinal e mortalidade infantil em Campina Grande, PB, Brasil. Revista Brasileira de Enfermagem, v.62, n.2, p.258-64, 2009. Disponível em < http://www.scielo.br/scielo.php?pid=S0034-

71672009000200014\&script=sci_abstract\&tlng=pt $>$. Acesso em: mar. 2017.

GBD 2015 Child Mortality Collaborators. Global, regional, national, and selected subnational levels of stillbirths, neonatal, infant, and under 5 mortality, 1980-2015: a systematic analysis for the Global Burden of Disease Study 2015. Lancet, v.388, p.1725-1774, 2016. Disponível em< http://thelancet.com/journals/lancet/article/PIIS0140-6736(16)31575-6/fulltext $>$. Acesso em: dez. 2016.

GONÇALVES, A. C. et al. Tendência da mortalidade neonatal na cidade de Salvador (BahiaBrasil), 1996-2012. Revista Brasileira de Saúde Materno Infantil, v.15, n.3, p.337-347, 2015. Disponível em < http://www.scielo.br/scielo.php?pid=S1519-

38292015000300337\&script=sci_arttext\&tlng=en>. Acesso em: mar. 2017. 
GUERRERO, A.V. P. Avaliabilidade do pacto pela redução da mortalidade infantil nas regiões Amazônia legal e nordeste do Brasil: descrição do programa e construção do modelo lógico. 2010. 74fl. Dissertação (Mestrado em Saúde Pública) - Centro de Pesquisa Aggeu Magalhães da Fundação Oswaldo Cruz, Recife, 2010.

IBGE. Instituto Brasileiro de Geografia e Estatística. Observações sobre a evolução da mortalidade no Brasil: o passado, o presente e perspectivas, 2010. Disponível em < https://www.ibge.gov.br/home/estatistica/populacao/tabuadevida/2009/notastecnicas.pdf> Acesso fev. 2017.

LISBOA, L. et al. Mortalidade infantil: principais causas evitáveis na região Centro de Minas Gerais, 1999-2011. Revista Epidemiologia Serviços de Saúde, v.24, n.4,p.711-720, 2015. Disponível em < http://www.scielo.br/pdf/ress/v24n4/2237-9622-ress-24-04-00711.pdf>. Acesso em: mar. 2017.

LOURENÇO, E.C; BRUNKEN, G.S;LUPPI, C.G. Mortalidade infantil neonatal: estudo das causas evitáveis em Cuiabá, Mato Grosso, 2007. Revista Epidemiologia Serviços de Saúde, v.22, n.4, p.697-706, 2013. Disponível em

<http://scielo.iec.gov.br/pdf/ess/v22n4/v22n4a16.pdf>. Acesso em: mar. 2017.

MALTA, D. C. et al. Lista de causas de mortes evitáveis por intervenções do Sistema Único de Saúde do Brasil. Revista Epidemiologia e Serviços de Saúde, v.16, n.4, p.233-244, 2007. Disponível em <

http://svs.aids.gov.br/dashboard/download/lista_causas_mortes_evitaveis.pdf >. Acesso em: mar. 2017.

MENEZES, S.T. et al. Classificação das mortes infantis em Belo Horizonte: utilização da lista atualizada de causas de mortes evitáveis por intervenções do Sistema Único de Saúde do

Brasil. Revista Brasileira de Saúde Materno Infantil, v.14, n.2, p.137-145, 2014.

Disponível em < http://www.scielo.br/scielo.php?pid=S1519-

38292014000200137\&script=sci_abstract\&tlng=pt>. Acesso em: mar. 2017.

NASCIMENTO, L. F; ALMEIDA, M.C; GOMES, C.M. Causas evitáveis e mortalidade neonatal nas microrregiões do estado de São Paulo. Revista Brasileira de Ginecologia Obstetrícia, v.36, n.7, p.303-9, 2014. Disponível em <

http://www.scielo.br/scielo.php?pid=S0100-72032014000700303\&script=sci_abstract $>$. Acesso em: mar. 2017.

OPAS. Organização Pan- Americana da Saúde. Conselho Diretor 55. Os objetivos de desenvolvimento do milênio e as meta de saúde: relatório final. 2016. Disponível em $<$ http://www.paho.org/hq/index.php?option=com_content\&view=article\&id=12276\%3A2016 -55th-directing-council-documents\&catid=8811\%3Adc-

documents\&Itemid=42078\&lang=en> . Acesso em: set. 2017.

OZA, S. et al. Neonatal cause of death estimates for the early and late neonatal periods for 194 countries: 2000-2013. Bulletin of the World Health Organization, v.93, n.1. p.19-28, 2015. Disponível em <http://researchonline.lshtm.ac.uk/2338154/>. Acesso em: dez 2016. 
RASELLA, D. Impacto do programa bolsa família e seu efeito conjunto com a estratégia saúde da família sobre a mortalidade no Brasil. 2013. 92fl. Tese (Doutorado em Saúde Pública) - Universidade Federal da Bahia, Salvador, 2013. Disponível em < https://repositorio.ufba.br/ri/handle/ri/12835>. Acesso em set. 2017.

RIPSA. Rede Interagencial de Informação para a Saúde Indicadores básicos para a saúde no Brasil: conceitos e aplicações. Brasília: Organização Pan-Americana da Saúde, 2008, n. 2, p.349. Disponível em < http://tabnet.datasus.gov.br/tabdata/livroidb/2ed/indicadores.pdf>. Acesso em: dez. 2017.

SILVA, Z. M. Estudo da notificação do óbito infantil em quatro municípios do estado do Piauí nos anos de 2005 e 2006. 2009. 63fl. Dissertação (Mestrado em Saúde Pública) Escola Nacional de Saúde Pública Sérgio Arouca, Rio de Janeiro, 2009. Disponível em < http://bdtd.ibict.br/vufind/Record/CRUZ_3ab3797403b805fb6f9255c2a4f06d1a>. Acesso em: dez. 2017.

WHO. WORLD HEALTH STATISTICS 2016: monitoring health for the SDGs, sustainable development goals. World Health Organization. WHO Library Cataloguing in Publication data, 2016. Disponível em <http://www.who.int/gho/publications/world_health_statistics/2016/en/>. Acesso em: set. 2017.

WHO. WORLD HEALTH STATISTICS 2014. World Health Organization. WHO Library Cataloguing in Publication data, 2014. Disponível em <http://www.who.int/gho/publications/world_health_statistics/2014/en/>. Acesso em: set. 2017. 